\title{
Safety and efficacy of non-anesthesiologist administration of propofol sedation during esophagogastroduodenoscopy in the intensive care unit
}

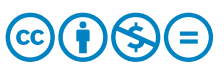

\author{
Authors \\ Janaki Patel, John Fang, Linda J. Taylor, Douglas G. Adler, Andrew J. Gawron
}

Institution

Department of Internal Medicine, Division of

Gastroenterology and Hepatology, University of Utah

School of Medicine, Salt Lake City, Utah, United States

submitted 30.8 .2018

accepted after revision 15.11 .2018

Bibliography

DOI https://doi.org/10.1055/a-0829-6284 |

Endoscopy International Open 2019; 07: E625-E629

(c) Georg Thieme Verlag KG Stuttgart · New York

ISSN 2364-3722

Corresponding author

Andrew J. Gawron, 30 N 1900 E, SOM 4R118, Salt Lake City, UT 84132

Fax: +1-801-581-7476

andrew.gawron@hsc.utah.edu

\section{ABSTRACT}

Background and study aims Propofol sedation is an increasingly popular method of sedation for gastrointestinal endoscopic procedures. The safety and efficacy of the nonanesthesiologist administration of propofol (NAAP) sedation has been demonstrated in the ambulatory setting. However, NAAP sedation in intensive care unit (ICU) patients has not been reported. The purpose of this study is to determine safety and efficacy of NAAP sedation in an ICU population.

Methods We retrospectively reviewed esophagogastroduodenoscopies (EGD) performed with NAAP sedation in our intensive care units from June 2014 to September 2016. All EGDs were performed for evaluation of gastrointestinal bleeding. The primary end point of this study was to analyze the incidence of sedation-related adverse events (AEs). The secondary end points included successful completion of procedure and any endoscopic interventions performed.

Results Two of 161 procedures (1.2\%) had sedation-related AEs requiring procedure termination. One hundred forty-six of 161 procedures $(90.7 \%$ ) were successfully completed. Incomplete procedures were due to excess heme, retained food or obstructive lesions (13/161, 8.1\%). Endoscopic intervention was performed successfully in $17 / 24$ cases $(70.8 \%)$ that had endoscopically treated lesions identified. One hundred six of 161 patients (66\%) were American Society of Anesthesiologists (ASA) classification III or IV.

Conclusion Our retrospective analysis demonstrated that EGDs can be successfully completed in ICU patients using NAAP sedation. When procedures cannot be completed, it is rarely due to sedation-related AEs. NAAP sedation further allows adequate examination and successful treatment of high-risk lesions. NAAP sedation appears safe and effective for endoscopic procedures in the ICU setting.

\section{Introduction}

Propofol (2,6-diisopropylphenol) sedation for endoscopic procedures is increasing in use $[1,2]$. Because propofol is highly lipophilic and crosses the blood-brain barrier quickly it has a rapid onset of action. In addition, its short half-life (2-4 minutes) results in rapid post-procedure recovery $[3,4]$. The ability to provide rapid and safe moderate sedation to perform endoscopic procedures has made it a highly desirable sedative option for both physicians and patients [5-7].
Propofol sedation, when administered under the guidance of a physician who is not an anesthesiologist, is defined as non-anesthesiologist administered propofol (NAAP). Propofol when used in combination with an opioid agent such as fentanyl is termed balanced propofol sedation (BPS) and results in optimal moderate sedation and amnestic effect allowing for thorough evaluation and endoscopic intervention [8]. A joint statement issued in 2009 by all the four US gastrointestinal professional societies stated "The administration of propofol and standard sedation by non-anesthesiologists is comparable with respect to their efficacy and safety profiles. Proper training and 
patient selection are crucial for the safe practice of NAAP sedation" [9].

Propofol sedation has been shown to be safe for numerous surgical procedures and for patients on mechanical ventilation. Initial reports of safe propofol sedation in ICU patients on mechanical ventilation were published over 30 years ago, including patients with higher American Society of Anesthesiologists risk class (ASA) [10-12]. More recent studies have established the safety and effectiveness of NAAP for both standard and advanced endoscopic procedures; these studies were performed in the outpatient setting in patients with relatively low ASA risk class and lower severity of illnesses [13-16]. There is greater concern regarding the safety of NAAP sedation for digestive endoscopy in hospitalized patients with a higher number of comorbidities and greater severity of illness [17].

Of significant importance, to date there have been no prior studies evaluating the safety of NAAP sedation for digestive endoscopy in hospitalized patients, especially patients admitted to an intensive care unit (ICU). This is a critical gap in knowledge regarding the safety of NAAP sedation in ICU patients undergoing endoscopy. Our institution routinely performs endoscopic procedures using NAAP in a variety of inpatient and outpatient settings. The aim of our study was to evaluate the safety and efficacy of non-anesthesiologist- administered Propofol (NAAP) sedation for esophagogastroduodenoscopies (EGDs) performed in acutely ill patients with gastrointestinal bleeding in an Intensive Care Unit (ICU) setting.

\section{Methods}

\section{Study design}

A retrospective chart review was performed on all EGDs performed in the medical, surgical, cardiovascular, and neurosurgical ICUs at a single, tertiary care referral center from June 2014 to September 2016 after obtaining Institutional Review Board approval.

\section{Inclusion/exclusion criteria}

Patients having an EGD for gastrointestinal bleeding using NAAP sedation in the ICU were included in our study. Patients were excluded if they were on mechanical ventilation or on vasopressor support (dopamine, dobutamine, epinephrine, norepinephrine, phenylephrine, or vasopressin). Patients on vasopressors or mechanical ventilation were excluded to avoid confounding results as our primary outcome measured was sedation related cardiovascular and pulmonary complications.

\section{NAAP sedation in EGDs performed in ICU}

NAAP was performed on all the procedures under endoscopist guidance and administered by the ICU nurses per our institutional protocol. ICU nurses and gastroenterology endoscopy providers at our institution are certified in advanced cardiac life support (ACLS) and Basic Life Support (BLS) and undergo a sedation training program (Safe Sedation Training, Applied Medical Visualizations) which includes airway measures, drug administration and rescuing patients from inadvertent deep sedation. Recertification is required every 2 years. New nursing staff are further required to administer sedation and documentation under supervision by a senior nurse prior to working independently.

Five people were present during endoscopy. Personnel present for all EGD procedures included two ICU nurses, an endoscopy lab technician, attending gastroenterologist, and a gastroenterology fellow per institutional policy. One nurse administered medications and the second nurse charted medications administered, vital signs, and any interventions including those performed for hemodynamic and respiratory decompensation. All patients were administered supplemental oxygen by nasal cannula per standard protocol. An initial induction dose of fentanyl usually $(12.5-25 \mathrm{mcg})$ and propofol $(20-30 \mathrm{mg})$ was given with subsequent administration of propofol of 10 to $30 \mathrm{mg}$ every 2 to 3 minutes to ensure adequate and sustained moderate sedation to allow completion of the procedure per the endoscopists' discretion. Patients had continuous pulse oximetry and continuous heart rate monitoring using a five-lead electrocardiogram monitor. Blood pressures were recorded every 2.5 to 3 minutes per institutional standard protocol. Promotility agents (e.g. erythromycin IV) are not routinely given prior to EGD procedures at our institution.

\section{Data sources and measures}

The institutional electronic medical record database (Epic Systems Corp., Madison, Wisconsin) and endoscopy electronic medical record database (ProVation Medical, Inc., Minneapolis, Minn) were queried for all EGDs performed in ICU patients over age 18 for this time period.

Demographic data collected included: gender, age, body mass index (BMI), date and time of hospital admission, date and time of ICU admission, history of significant co-existing respiratory disease (i.e. pulmonary fibrosis, chronic obstructive pulmonary disease, lobectomies, obstructive sleep apnea, asthma, and chronic hypoxemia), any baseline oxygen requirement, procedure date and duration. Laboratory data included hemoglobin, hematocrit, international normalized ratio (INR), units of fresh frozen plasma, packed red blood cells, and platelets transfused prior to the procedure.

Procedural data recorded included: ASA classification, heartrate, oxygen saturation, blood pressure, and total doses of propofol and fentanyl. Adverse events (AEs) were defined as hypotension when systolic blood pressure $<90 \mathrm{mmHg}$, bradycardia when heartrate $<60$ beats per minute (BPM) and oxygen desaturation when pulse oximetry $<90 \%$. Airway interventions reviewed included: use of nasopharyngeal or oropharyngeal airway, non-rebreather mask, bag-mask ventilation, noninvasive ventilation with bi-level positive airway pressure (BiPAP) or need for endotracheal intubation.

Other procedural data collected included: procedure indication, findings and any endoscopic interventions that were performed. Lesions were identified as endoscopically treatable according to American College of Gastroenterology (ACG) gastrointestinal ulcer guidelines [18]. Esophageal varices with stigmata of recent bleeding such as fibrin clots of red wale signs were also included as requiring endoscopic treatment. Endoscopic intervention performed was collected as epinephrine injection, 


\begin{tabular}{|l|r|}
\hline \begin{tabular}{l} 
Table 1 EGD Procedural characteristics. \\
\hline Total procedure time in minutes (mean \pm SD)
\end{tabular} & $15.5 \pm 11.0$ \\
\hline Total propofol dose $(\mathrm{mg})($ mean \pm SD) & $145.9 \pm 93.1$ \\
\hline Total fentanyl dose $(\mathrm{mcg})($ mean $\pm \mathrm{SD})$ & $36.5 \pm 22.5$ \\
\hline High-risk lesions $(\mathrm{N}, \%)$ & $24(14.9 \%)$ \\
\hline Intervention performed $(\mathrm{N}, \%)$ & $17(70.8 \%)$ \\
\hline Successfully completed $(\mathrm{N}, \%)$ & $146(90.6 \%)$ \\
\hline Incomplete procedure $(\mathrm{N}, \%)$ & \\
\hline " Presence of heme & $7(4.3 \%)$ \\
\hline - Food in the stomach & $2(1.2 \%)$ \\
\hline - Obstructive ulcer & $2(1.2 \%)$ \\
\hline - Hemodructive mass & $2(1.2 \%)$ \\
\hline EGD, esophagogastroduodenoscopy; SD, standard deviation \\
\hline
\end{tabular}

application of hemostatic clips, bipolar cauterization, and variceal banding. A procedure was also defined as successfully completed if the second portion of the duodenum (or jejunum in the case of previous gastric surgery) was reached. The specific reasons for incomplete procedures were also collected and summarized in $>$ Table 1.

\section{Outcomes}

The primary outcome was occurrence of any sedation-related AEs such as hypoxia, bradycardia, hypotension, or airway intervention as defined above. Secondary outcomes were defined as successful endoscopic intervention if performed and successful completion of procedure.

\section{Statistical analysis}

Descriptive statistics were performed for patient demographic and clinical characteristics. AEs are reported as proportions of the entire sample. All statistical analyses were performed using STATA 12.0 (StatCorp LLC, College Station, Texas, United States).

\section{Results}

A total of 161 patients' procedure records out of 239 procedures met inclusion criteria and had complete data available for extraction during the study period. Patient characteristics are reported in $>$ Table 2 . Most patients were ASA III (56.5\%). $32.3 \%$ were ASA II, $9.3 \%$ were ASA IV and only $1.9 \%$ were categorized as ASA I. Average patient age was 60.2 years ( \pm 14.0 ) with BMI 27.6 ( \pm 6.5$)$. The average hemoglobin value prior to the procedure was $8.5 \mathrm{~g} / \mathrm{dL}( \pm 2.0)$. A total of $26 / 161(16.2 \%)$ had underlying respiratory disease and 104/161 (64.6\%) had baseline requirement of supplemental oxygen. Gastrointestinal bleeding indications for the procedures were classified as hematemesis $37.2 \%$, coffee ground emesis $8.7 \%$, melena $51.5 \%$, hematochezia $18.6 \%$, anemia, $9.9 \%$ or history of ulcer $1.2 \%$.

The primary outcome of sedation-related AEs is summarized in $>$ Table 3 . Only two procedures $(1.2 \%)$ were terminated due
- Table 2 Patient characteristics.

\begin{tabular}{|l|c|}
\hline Age (Mean \pm SD) & $60.2 \pm 14.0$ \\
\hline BMI (Mean \pm SD) & $27.6 \pm 6.5$ \\
\hline $\begin{array}{l}\text { Sex (N,\%) } \\
\text { - Male }\end{array}$ & $102(63.4 \%)$ \\
\hline - Female & $59(36.6 \%)$ \\
\hline $\begin{array}{l}\text { ASA classification (N,\%) } \\
\text { - ASA I }\end{array}$ & $3(1.9 \%)$ \\
\hline - ASA II & $52(32.3 \%)$ \\
\hline - ASA III & $91(56.5 \%)$ \\
\hline - ASA IV & $15(9.3 \%)$ \\
\hline $\begin{array}{l}\text { Past medical history of underlying respiratory } \\
\text { disease (N,\%) }\end{array}$ & $26(16.2 \%)$ \\
\hline \begin{tabular}{l} 
Patient requiring baseline oxygen (N,\%) \\
\hline Hemoglobin prior to the procedure (mean \pm SD)
\end{tabular} & $8.5 \pm 2.0$ \\
\hline INR prior to the procedure (mean \pm SD) & $104(64.6 \%)$ \\
\hline $\begin{array}{l}\text { Transfusion history prior to the procedure } \\
\text { (mean [min - max] units) }\end{array}$ & \\
\hline - PRBC & \\
\hline - Platelets & $1.6 \pm 0.7$ \\
\hline - Fresh frozen plasma & $0.1(0-4)$ \\
\hline $\begin{array}{l}\text { SD, standard deviation; BMI, body mass index; ASA, American Society of } \\
\text { Anesthesiologists; INR, international normalized ratio; PRBC, packed red } \\
\text { blood cells }\end{array}$ \\
\hline
\end{tabular}

- Table 3 Sedation-related adverse events.

Hypoxia

- Lowest oxygen desaturation (mean $\pm \mathrm{SD}$ )

$97.2 \pm 3.8$

- $<90 \%(\mathrm{~N}, \%)$

$12(7.5 \%)$

Airway interventions ( $N, \%$ )

- Intubation

$0(0 \%)$

- Non-rebreather masks

$8(4.97 \%)$

- BiPAP ${ }^{1}$

$2(1.24 \%)$

Hypotension

- Lowest systolic BP (mean \pm SD)

- Lowest diastolic BP (mean \pm SD)

$104.7 \pm 18.2$

- $<90 / 60(\mathrm{~N}, \%)$

Bradycardia

- Lowest HR (mean \pm SD)

$86.0 \pm 14.9$

- <60 BPM (N,\%)

$8(5.0 \%)$

BiPAP, bilevel positive airway pressure; BP, blood pressure; HR, heart rate; SD, standard deviation; BPM, beats per minute

${ }^{1}$ Required respiratory therapist to administer

to sedation-related AEs, one secondary to hypotension (systolic blood pressure $<80$ ) and the other due to increased requirement for sedation due to patient's agitation. On average, oxygen saturation was $97.2 \%( \pm 3.8 \%)$ with documented desaturation in 12 patients (7.5\%). Interventions to address sedationrelated adverse respiratory events included: use of non-rebreather masks in eight patients (5.0\%) and use of BiPAP in two patients (1.2\%). No patients required bag-mask ventilation or endotracheal intubation for mechanical ventilation. No procedures were terminated due to hypoxia. On average, systolic 
blood pressure was 104.7 ( \pm 18.2) $\mathrm{mm} \mathrm{Hg}$ and diastolic blood pressure $59.8( \pm 12.7) \mathrm{mmHg}$. Average heart rate during procedures was 86 ( \pm 14.9 ) BPM. Transient episodes (<3 minutes) of hypotension $(23 \%)$ or bradycardia $(5.0 \%)$ were addressed by delaying the next administered propofol dose.

Success of endoscopic treatment to achieve hemostasis is summarized in Table1. Twenty-four of 161 procedures (15\%) had lesions identified with high-risk stigmata of recent bleeding such as: Forrest classification la (active spurting), Ib (active oozing), Ila (visible vessel) and IIb (adherent clot). Successful hemostasis was achieved in 17 of 24 procedures (70.8\%). Hemostasis was unable to be achieved in the seven procedures due to findings of mature clot (4 procedures), unable to perform intervention due to friable mucosa (1 procedure), and difficult location with no active bleeding (2 procedures).

Successful completion of the procedure was obtained in 146 of 161 procedures $(90.6 \%)$. Thirteen of the 15 (9.3\%) incomplete procedures were due to either presence of food or blood precluding adequate visualization or obstructive mass or ulcers preventing further advancement. On average, procedures were 15.5 ( \pm 11.0$)$ minutes long requiring $145.9 \mathrm{mg}( \pm 93.1)$ of propofol and $36.5 \mathrm{mcg}( \pm 22.5)$ of fentanyl. Two procedures were performed using midazolam; one was with propofol and fentanyl and another with midazolam and fentanyl.

\section{Discussion}

EGDs performed in an ICU setting are often performed in patients with significant comorbidities and increased acuity of illness as reflected in their higher ASA score [19].These factors place them at higher risk for hemodynamic instability and cardio-respiratory complications from sedation [20,21]. Our study is the first report on the safety and outcomes of NAAP sedation for EGD in patients admitted to the ICU for management of gastrointestinal bleeding. Our results suggest that EGDs can be safely and adequately completed with NAAP sedation in patients admitted to an ICU for gastrointestinal bleeding.

Overall, sedation-related AEs in patients in the ICU undergoing EGD for bleeding who were sedated via NAAP were uncommon, transient, and rapidly reversed without requiring termination of the procedure in nearly all patients ( $\triangleright$ Table 2 ). Most cardiovascular events such as hypotension and bradycardia resolved after increasing the interval between the subsequent propofol administrations per our usual clinical practice. Desaturations were reversed using interventions such as use of non-rebreather mask or rarely use of BiPAP. None of the procedures required additional aid such as bag-mask ventilation or endotracheal intubation. There was no obvious correlation to the amount of sedation administered to hemodynamic and respiratory compromise.

There are ample data on safety and efficacy of NAAP sedation when used in outpatient settings for routine endoscopic procedures [22,23]. NAAP sedation has also been found to be safe for advanced interventional endoscopic procedures including in an elderly population with relatively high comorbidities [24]. Our data support the relative safety and efficacy of NAAP sedation for EGD procedures in ICU patients. Our institution has a structured training program for propofol administration when performed by non-anesthesia personnel to ensure endoscopists and nurses are comfortable with administering sedation and handling sedation-related AEs. A study from Denmark has also demonstrated the safety of NAPS after undergoing a structured training program including airway management by both nurses and endoscopists [25].

In 2015 the European Society of Gastrointestinal Endoscopy and the European Society of Gastroenterology and Endoscopy Nurses and Associates issued an updated guideline on non-anesthesiologist administration of propofol stating that NAAP can be administered by personnel who are not involved in the other parts of the procedure and the patient is continuously monitored. However, the ESGE guidelines further states that in the presence of risk factors such as ASA $\geq 3$, Mallampati class of 3 or conditions increasing the risk of airway compromise, an anesthesiologist must be present [26]. The 2018 American Society for Gastrointestinal Endoscopy (ASGE) guidelines state that, "NAPS (nurse administered Propofol sedation) should be administered by personnel specifically trained in its administration who should have expertise in emergency airway management and must be present continuously during its use". It also states that anesthesia provider-administered sedation be considered for complex endoscopic procedures or patients with multiple medical comorbidities or at risk for airway compromise but does not stratify risk by ASA class [27]. Our data demonstrate that NAAP can be safely administered in the ICU setting without an anesthesiologist to patients with ASA classification $\geq 3$ undergoing endoscopy.

\section{Limitations}

This study is a retrospective case series and there are limiting factors related to its retrospective design and lack of a control group. The study design does not allow us to draw conclusions about dose response or the effect of specific doses on patient outcomes. Excluding patients on mechanical ventilation and vasopressor support was important to measure the study outcomes so as not to mask sedation-related AEs. Vital signs including blood pressure were only charted at 2.5- to 3-minute intervals though continuous monitoring of oxygen saturation and heart rate was performed. Therefore, very transient episodes of hypoxemia or bradycardia may have not been recorded. In addition, a second ICU nurse was present during all procedures solely to chart medications given and procedural notes, making missing any significant $A E$ recording very unlikely. Fifteen percent of procedures at our institution identified high-risk lesions; these lesions can lengthen endoscopy and anesthesia duration (due to need for therapy). Thus, the results may not be generalizable to institutions with a higher incidence of high-risk lesions seen on EGD. Finally, this study was performed at a tertiary care institution where NAAP sedation is the primary method of sedation in both the ambulatory and ICU setting, thus there is a high level of expertise among endoscopists performing procedures with NAAP sedation, which may not be applicable in settings where endoscopists are not experienced with NAAP. 


\section{Conclusion}

This is the first study describing the safety and efficacy of NAAP sedation for EGD procedures performed in an ICU. We found that NAAP sedation can be administered safely and effectively in critically ill patients with a very low incidence of AEs and high completion rates when the non-anesthesia personnel are adequately trained and experienced. Larger, prospective, randomized studies in the ICU setting are warranted to compare NAAP sedation to other forms of sedation for ICU patients undergoing digestive endoscopy.

\section{Competing interests}

None

\section{References}

[1] Crespo J, Terán Á. Endoscopy and sedation: an inseparable binomia for the gastroenterologist. Rev Española Enfermedades Dig 2018; 110: $250-252$

[2] Pérez-Cuadrado-Robles E, Ferreira A, García-Cano ]. Enhancing the current evidence on endoscopist-directed propofol-based sedation. Rev Española Enfermedades Dig 2018; 110: 215-216

[3] Triantafillidis JK, Merikas E, Nikolakis D et al. Sedation in gastrointestinal endoscopy: Current issues. World J Gastroenterol 2013; 19: 463

[4] Folino T, Parks LPropofol. StatPearls Publishing; 2017: Available at: http://www.ncbi.nlm.nih.gov/pubmed/28613634

[5] Ng JM, Kong CF, Nyam D. Patient-controlled sedation with propofol for colonoscopy. Gastrointest Endosc 2001; 54: 8-13

[6] Cohen LB, Hightower CD, Wood DA et al. Moderate level sedation during endoscopy: a prospective study using low-dose propofol, meperidine/fentanyl, and midazolam. Gastrointest Endosc 2004; 59: $795-803$

[7] Uzman S, Gurbulak B, Gurbulak EK et al. A comparison of propofol and midazolam/meperidine sedation in upper gastrointestinal endoscopy. Wideochir Inne Tech Maloinwazyjne 2016; 11: 178-185

[8] VanNatta ME, Rex DK. Propofol alone titrated to deep sedation versus propofol in combination with opioids and/or benzodiazepines and titrated to moderate sedation for colonoscopy. Am J Gastroenterol 2006; 101: 2209-2217

[9] Vargo JJ, Cohen LB, Rex DK et al. Position statement: Nonanesthesiologist administration of propofol for Gl endoscopy. Hepatology 2009; 50: $1683-1689$

[10] Newman LH, McDonald JC, Wallace PG et al. Propofol infusion for sedation in intensive care. Anaesthesia 1987; 42: 929-937

[11] Marinella MA. Propofol for sedation in the intensive care unit: essentials for the clinician. Respir Med 1997; 91: 505-510
[12] Grounds RM, Lalor JM, Lumley J et al. Propofol infusion for sedation in the intensive care unit: preliminary report. Br Med J (Clin Res Ed) 1987; 294: $397-400$

[13] McVay T, Fang JC, Taylor L et al. Safety analysis of bariatric patients undergoing outpatient upper endoscopy with non-anesthesia administered propofol sedation. Obes Surg 2017; 27: $1501-1507$

[14] Yusoff IF, Raymond G, Sahai AV. Endoscopist administered propofol for upper-GI EUS is safe and effective: a prospective study in 500 patients. Gastrointest Endosc 2004; 60: 356-360

[15] Horiuchi A, Nakayama Y, Hidaka N et al. Low-dose propofol sedation for diagnostic esophagogastroduodenoscopy: results in 10,662 adults. Am J Gastroenterol 2009; 104: 1650 - 1655

[16] Gouda B, Gouda G, Borle A et al. Safety of non-anesthesia provider administered propofol sedation in non-advanced gastrointestinal endoscopic procedures: A meta-analysis. Saudi J Gastroenterol 2017; 23: $133-143$

[17] Rex DK, Overley CA, Walker ]. Registered nurse-administered propofo sedation for upper endoscopy and colonoscopy: Why? When? How? Rev Gastroenterol Disord 2003; 3: 70 - 80 [cited 2018 May 1]

[18] Laine L, Jensen DM. Management of patients with ulcer bleeding. Am J Gastroenterol 2012; 107: 345-360

[19] Lupei MI, Chipman JG, Beilman G] et al. The association between ASA status and other risk stratification models on postoperative intensive care unit outcomes. Anesth Analg 2014; 118: 989-994

[20] Hopkins TJ, Raghunathan K, Barbeito A et al. Associations between ASA Physical Status and postoperative mortality at $48 \mathrm{~h}$ : a contemporary dataset analysis compared to a historical cohort. Perioper Med (London, England) 2016; 5: 29

[21] Uzman S, Yilmaz $Y$, Toptas $M$ et al. A retrospective analysis of postoperative patients admitted to the intensive care unit. Hippokratia 2016; $20: 38-43$

[22] Sathananthan D, Young E, Nind G et al. Assessing the safety of physician-directed nurse-administered propofol sedation in low-risk patients undergoing endoscopy and colonoscopy. Endosc Int Open 2017; 5: E110-E115

[23] Sieg A, Beck S. bng-Study-Group. et al. Safety analysis of endoscopist-directed propofol sedation: a prospective, national multicenter study of 24441 patients in German outpatient practices. J Gastroenterol Hepatol 2014; 29: 517 - 523

[24] Schilling D, Rosenbaum A, Schweizer S et al. Sedation with propofol for interventional endoscopy by trained nurses in high-risk octogenarians: a prospective, randomized, controlled study. Endoscopy 2009; 41: $295-298$

[25] Jensen JT, Vilmann P, Horsted T et al. Nurse-administered propofol sedation for endoscopy: a risk analysis during an implementation phase. Endoscopy 2011; 43: 716-722

[26] Dumonceau J-M, Riphaus A, Schreiber F et al. Non-anesthesiologist administration of propofol for gastrointestinal endoscopy: European Society of Gastrointestinal Endoscopy, European Society of Gastroenterology and Endoscopy Nurses and Associates Guideline - Updated June 2015. Endoscopy 2015; 47: 1175-1189

[27] Early DS, Lightdale JR et al. ASGE Standards of Practice Committee. GUIDELINE Guidelines for sedation and anesthesia in GI endoscopy. Gastroinest Endosc 2018; 87: 327-337 